\title{
Robust Mesh Deformation using the Linear Elasticity Equations
}

\author{
Richard P. Dwight \\ Institute of Aerodynamics and Flow Technology, German Aerospace Center \\ (DLR), D-38108 Braunschweig, Germany. richard.dwight@dlr.de
}

Summary. A modification is proposed to the equations of linear elasticity as used to deform Euler and Navier-Stokes meshes. In particular it is seen that the equations do not admit rigid body rotations as solutions, and it is shown how these solutions may be recovered by modifying the constitutive law. The result is significantly more robust to general deformations, and combined with incremental application generates valid meshes well beyond the point at which remeshing is required.

\section{Introduction}

The ability to deform meshes according to changes in the computational domain is a fundamental tool in CFD, and a foundation on which many other processes are built. It is for example essential in aerodynamic optimization, where remeshing the geometry after each design step would cause the change in the quantity of interest to be swamped by the discretization error [3]. Achieving high-order accuracy in time is much easier when the mesh topology remains unchanged between time steps [5], but grid deformation is commonly used in a wide variety of other applications simply because it tends to be considerably cheaper and more convenient than remeshing; an example is aero-elastic coupling.

However in practice most mesh deformation algorithms suffer from robustness problems when faced with large deformations (in particular those involving large relative motion of bodies), but poor quality input meshes cause difficulties for small deformations too. Even worse many important problems involve these two aspects combined; a particularly acute example is the movement of the flaps and slats of 3d high-lift configurations, for which few algorithms perform satisfactorily.

One method that shows considerable promise models the mesh as an elastic solid using the equations of linear elasticity. It was applied in [1], together with refinement and derefinement for time-dependent problems undergoing substantial deformation. Stein et al. [4] have applied it with an elastic stiffness varying in inverse proportion to cell volume thereby preserving quality in boundary-layers and regions of high resolution, and in [5] this modification was seen to be considerably more robust than several other deformation algorithms tested.

Here the equations are discretized using a Galerkin finite element method with piecewise linear shape-functions on triangular and tetrahedral unstructured grids. A novel modification is developed by examining the behaviour of the equations under rigid rotations of the mesh, and modifying the equations to admit these solutions. The modified equations are shown to be much more robust to large deformations. By 
applying the deformation incrementally, extremely large displacements are shown for $2 \mathrm{~d}$ and 3d Navier-Stokes (NS) meshes.

\section{Governing Equations}

The equations of linear elasticity govern small displacements $u(x)=(u, v, w)$ of an elastic solid subject to body forces and surface tractions. Using the summation convention they may be written

$$
\nabla \cdot \sigma=f \quad \text { on } \Omega,
$$

where $f$ is some body force, $\Omega$ is the computational domain, and $\sigma$ is the stress tensor, given in terms of the strain tensor $\epsilon$ by the constitutive relation

$$
\sigma=\lambda \operatorname{Tr}(\epsilon) I+2 \mu \epsilon
$$

where $\operatorname{Tr}$ is the trace, $\lambda$ and $\mu$ are the Lamé constants, and are a property of the elastic material. It is convenient to express these in terms of Young's modulus $E$ and Poisson's ratio $\nu$ as

$$
\lambda=\frac{\nu E}{(1+\nu)(1-2 \nu)}, \quad \mu=\frac{E}{2(1+\nu)} .
$$

$E>0$ may be thought of as the stiffness of the material, where large $E$ indicates rigidity. Poisson's ratio $\nu$ is a measure of how much the material shrinks in the lateral direction as it extends in the axial direction; for physical materials $-1<\nu<\frac{1}{2}$. The linear kinematic law

$$
\epsilon=\frac{1}{2}\left(\nabla u+\nabla u^{T}\right),
$$

quantifies the change in length and orientation of a material fibre in the elastic body. Boundary conditions are Dirichlet, $u=g$ on $\partial \Omega$, completing the system.

A significant advantage of the application of these equations for mesh deformation is that diverse features required in practice can be readily and simply realized in a manner consistent with the model of the mesh as an elastic body. For example for a symmetry plane it is convenient that mesh points move, but remain within the plane, which may be implemented by the specification of only one coordinate of the displacement. For adaptive methods it may be desirable to deform the mesh such as to cluster mesh points in regions of interest, which is readily possible by applying a non-zero body force $f$.

\section{Finite Element Discretization}

The governing equations are discretized on a triangular or tetra Euler or NS mesh using a Galerkin method based on the trial and test spaces

$$
\begin{aligned}
U^{h} & =\left\{u^{h} \mid u^{h} \in H^{h}(\Omega)^{n}, u^{h}=g^{h} \text { on } \partial \Omega\right\}, \\
\Phi^{h} & =\left\{\phi^{h} \mid \phi^{h} \in H^{h}(\Omega)^{n}, \phi^{h}=0 \text { on } \partial \Omega\right\},
\end{aligned}
$$


where $H^{h}(\Omega)$ is a finite-dimensional function space on $\Omega$, and $n$ is the dimension of the space. The finite element problem may then be stated: find $u^{h} \in U^{h}$ such that $\forall \phi^{h} \in \Phi^{h}$

$$
\int_{\Omega} \epsilon\left(\phi^{h}\right): \sigma\left(u^{h}\right) \mathrm{d} \Omega=\int_{\Omega} \phi^{h} \cdot f \mathrm{~d} \Omega .
$$

It is expected that the mesh resolution will be more than adequate to resolve features of the elastic solution, therefore $H^{h}$ is taken as the space of functions linear on the elements of the grid, and then $\epsilon\left(\phi^{h}\right): \sigma\left(u^{h}\right)$ is constant on the elements, simplifying implementation considerably.

The linear system is solved using an ILU $(m)$ preconditioned restarted GMRES method, with a restart vector of 50, using the PETSc library [2]. For high-Reynolds number NS grids, an ILU fill-in of $m=4$ is required for convergence, leading to high memory costs relative to the flow solver.

\section{Admission of Rigid-Body Rotations}

While accuracy per se is not of concern, it seems reasonable to request that the deformation equations admit rigid body motions of the mesh, which is not true for the linearized kinematic law (4), which gives a non-zero strain for a rotation. An improvement, such as the Lagrangian strain tensor,

$$
\epsilon=\frac{1}{2}\left(\nabla u+\nabla u^{T}+\nabla u^{T} \cdot \nabla u\right)
$$

must necessarily be non-linear, raising the computational cost of the method unacceptably. However, although the linear strain is non-zero for a rotation (and for any affine transformation) it is independent of $x$ :

$$
u=\left[\left(\begin{array}{cc}
\cos \theta & \sin \theta \\
-\sin \theta & \cos \theta
\end{array}\right)-I\right] \cdot x, \quad \epsilon=\left(\begin{array}{cc}
\cos \theta-1 & 0 \\
0 & \cos \theta-1
\end{array}\right),
$$

and therefore the stress is also independent of $x$, equation (1) is satisfied, and rigid body rotations are admitted "by accident".

However varying the stiffness of grid cells based on their size increases the robustness of the method to large deformations considerably. But this introduces an $x$ dependence into the constitutive law, and rigid body rotations are lost as solutions. To recover them, it is sufficient that $\sigma=0$ for rotations. Substituting the expression for $\epsilon$ above into (2) we have

$$
\sigma=2 \lambda(\cos \theta-1) I+2 \mu(\cos \theta-1) I=(\lambda+\mu)[2(\cos \theta-1)] I,
$$

which may be set to zero by choosing $\lambda+\mu=0$. This is achieved by replacing the expressions of (3) by $\lambda=-E, \mu=E$. The same effect can be obtained by setting the Poisson ratio $\nu$ to a very large value, which emphasizes that the equations can no longer be thought of as a model of elasticity, although they behave somewhat similarly. Effectively a defect in the kinematic relation has been corrected by introducing a defect into the constitutive law, resulting in an entirely new set of equations. Nonetheless in the following section it will be seen that not only are rigid body rotations now admitted, but that the scheme is mush more robust to other deformations. 


\section{Numerical Results}

Two two-dimensional test cases are considered: a Euler grid for a NACA0012 single element aerofoil with a coarse fully unstructured Euler grid, a circular farfield at a distance of 40 chord lengths, and the aerofoil is rotated about its nose while the farfield is held stationary, see e.g. Figure 2. Also a NS grid about a three-element high-lift configuration where the slat is deflected and the remaining elements held fixed, see Figure 1. The robustness of the modified equations is compared with that of the standard equations for $-1<\nu<0.5$, by determining the maximum deflection that still results in a valid grid, with no negative volumes. In all cases $E$ is proportional to the inverse of the cell volume. The results are shown on the left of Figure 1; the modified constitutive law allows a $70 \%$ greater rotation of the NACA, and a $40 \%$ greater deflection of the flap. The grid for the greatest valid flap deflection is shown on the right of Figure 1. Near the body the point distributions are almost unmodified due to the much greater elastic stiffness there, a desirable property given that the initial meshes are likely to resolve surface regions well.

Provided intermediate surface definitions exist, exceptionally large deformations are possible if several steps are taken. For example for the NACA0012 by first calculating the grid for a $90^{\circ}$ turn an additional $90^{\circ}$ rotation was possible. Figure 2 shows the NACA rotated completely 4 times, the left-hand plot shows the rotation angle against the smallest angle in the grid (a measure of grid quality) for several increment sizes. The same may be done for the high-lift case, resulting in a valid grid for a flap deflection of $155^{\circ}$, the tip of the flap almost touching the main element, and an entire grid block compressed into the intervening space, Figure 3. Of course a flow solution on this grid reveals defects in the solution, notably the black triangle of low pressure at the trailing edge of the main element.

Finally Figure 4 shows the deformation of a $3 d$ turbulent wing-body configuration mesh, whereby the calculation was performed in two steps, a deflection first to $45^{\circ}$ and then to $90^{\circ}$. The mesh close to the wing is almost unchanged; because of the relatively rapid grid coarsening with distance from the surface, the algorithm is able to put the weight of the deformation onto exclusively large cells.

\section{Conclusions and Further Work}

It has been shown that it is possible to build deformation algorithms that allow extremely large deformations of poor quality meshes, something that is not taken for granted in the aerospace community. It has also been seen that the linear elastic method may be made significantly more robust with a minor modification to the governing equations. The efficient solution of these equations requires some study; the current ILU preconditioned GMRES approach requires excessive storage in 3d, and a Jacobi iteration converges extremely slowly for NS grids. Since by far the dominant source of stiffness is the high aspect-ratio cells in the boundary-layer a line-implicit algorithm combined with multigrid would be particularly appropriate [5]. A second concern is the quality of the resulting meshes, which is not usually an issue for small deformations, but - as seen above - strongly deformed meshes are often not suitable for flow calculations. The amount by which a grid cell is stretched during a deformation could be quantified by examining the displacement 
Jacobians, and mesh refinement could be performed based on this indicator [1]. Finally a demanding practical test case should be considered.
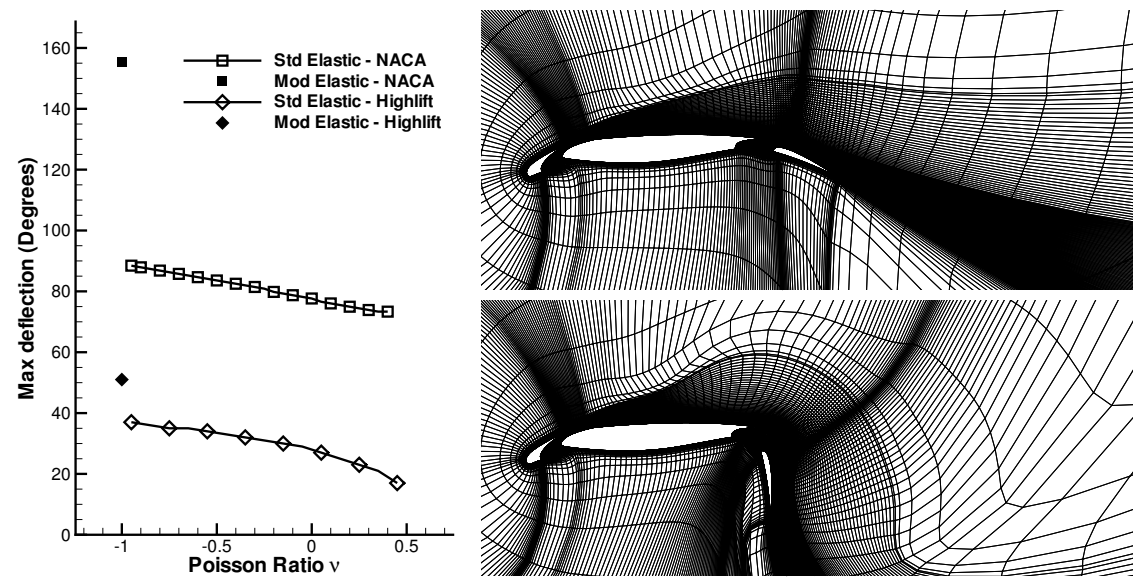

Fig. 1. Maximum possible deflection for a single step of the standard and modified elastic models for the NACA0012 and high-lift configurations. Initial and $51^{\circ}$ defected high-lift grids.
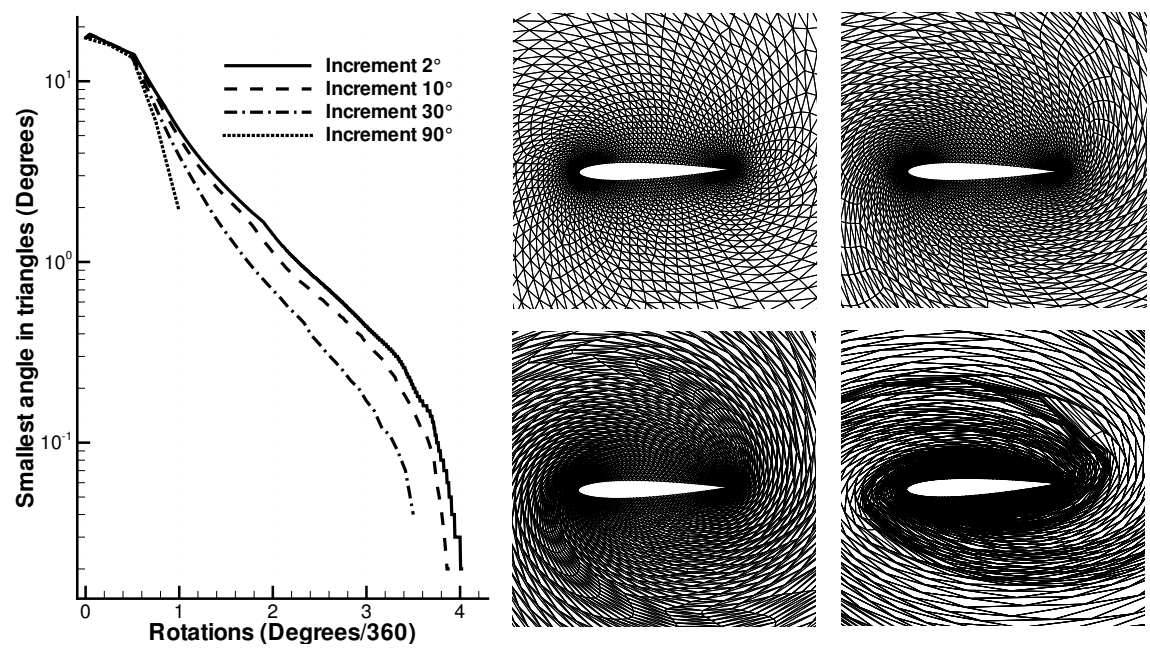

Fig. 2. Incremental rotation of a NACA0012 with steps of $2-90^{\circ}$. 

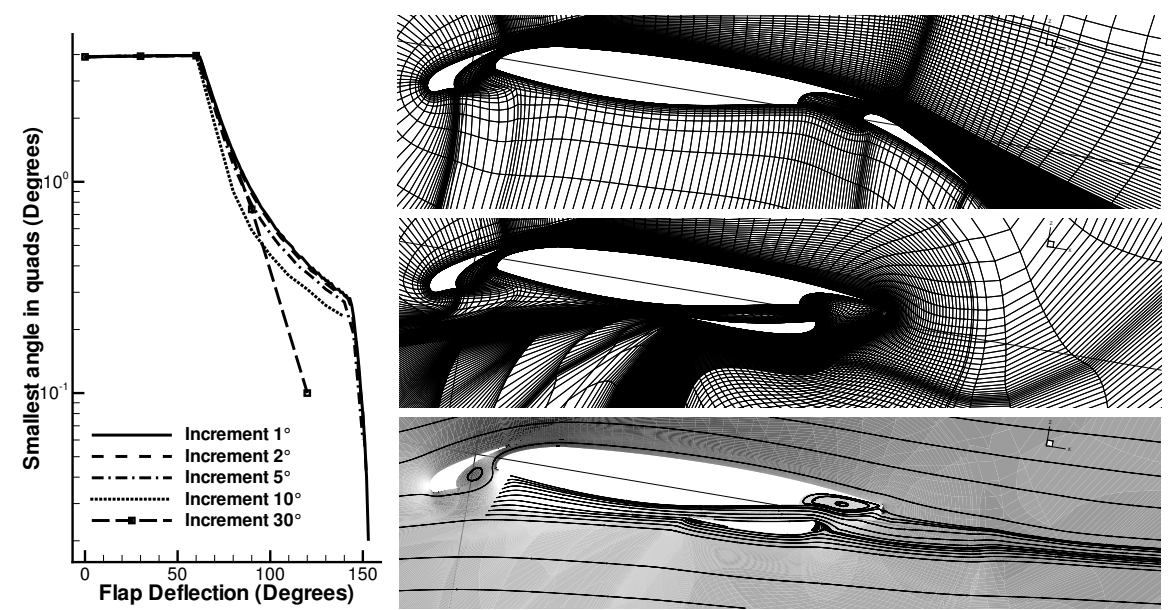

Fig. 3. Incremental deflection of flap to $150^{\circ}$.

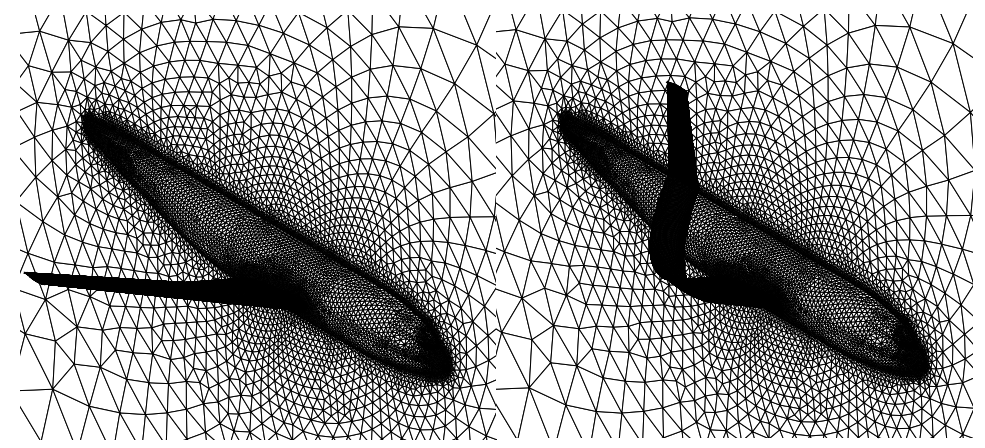

Fig. 4. Deformed mesh for the DLR-F4 wing-body configuration.

\section{References}

1. T. Baker and P. Cavallo. Dynamic adaption for deforming tetrahedral meshes. 37th AIAA Aerospace Sciences Meeting and Exhibit, January, Reno, NV. AIAA1999-3253, 1999.

2. S. Balay, K. Buschelman, W.D. Gropp, D. Kaushik, M.G. Knepley, L. CurfmanMcInnes, B.F. Smith, and H. Zhang. PETSc Web page, 2006. http://www.mcs.anl.gov/petsc.

3. R.P. Dwight and J. Brezillon. Effect of various approximations of the discrete adjoint on gradient-based optimization. Proceedings of the 44th AIAA Aerospace Sciences Meeting and Exhibit, Reno NV. AIAA-2006-0690, 2006.

4. K. Stein, T. Tezduyar, and R. Benney. Mesh moving techniques for fluidstructure interactions with large displacements. Journal of Applied Mechanics, 70:58-63, 2003.

5. Z. Yang and D. Mavriplis. Unstructured dynamic meshes with higher-order time integration schemes for the unstready Navier-Stokes equations. 43th AIAA 
Aerospace Sciences Meeting and Exhibit, January 10-13, Reno, NV. AIAA-20051222, 2005 